\title{
Effects of sleep deprivation on different phases of memory in the rat: dissociation between contextual and tone fear conditioning tasks
}

\section{Vanessa Contatto Rossi ${ }^{1}$, Paula Ayako Tiba ${ }^{2}$, Karin Di Monteiro Moreira' ${ }^{2}$, Tatiana Lima Ferreira ${ }^{2}$, Maria Gabriela Menezes Oliveira ${ }^{1}$ and Deborah Suchecki ${ }^{1}$ *}

' Department of Psychobiology, Escola Paulista de Medicina, Universidade Federal de São Paulo - UNIFESP, São Paulo, Brazil

${ }^{2}$ Centro de Matemática, Computação de Cognição, Universidade Federal do ABC - UFABC, Santo André, Brazil

\section{Edited by:}

Benno Roozendaal, Radboud

University Nijmegen Medical

Centre, Netherlands

\section{Reviewed by:}

Ted Abel, University of

Pennsylvania, USA

Monika R. Fleshner, University of

Colorado, USA

\section{${ }^{*}$ Correspondence:}

Deborah Suchecki, Department of Psychobiology, Escola Paulista de Medicina, Universidade Federal de São Paulo - UNIFESP, Rua Napoleão de Barros 925, São Paulo, SP 04024-002, Brazil

e-mail: deborah.suchecki21@

unifesp.br;

deborah.suchecki@gmail.com
Numerous studies show that sleep deprivation (SD) impacts negatively on cognitive processes, including learning and memory. Memory formation encompasses distinct phases of which acquisition, consolidation and retrieval are better known. Previous studies with pre-training SD induced by the platform method have shown impairment in fear conditioning tasks. Nonetheless, pre-training manipulations do not allow the distinction between effects on acquisition and/or consolidation, interfering, ultimately, on recall of/performance in the task. In the present study, animals were first trained in contextual and tone fear conditioning (TFC) tasks and then submitted to SD with the purpose to evaluate the effect of this manipulation on different stages of the learning process, e.g., in the uptake of (new) information during learning, its encoding and stabilization, and the recall of stored memories. Besides, we also investigated the effect of SD in the extinction of fear memory and a possible state-dependent learning induced by this manipulation. For each task (contextual or TFC), animals were trained and then distributed into control, not sleep-deprived (CTL) and SD groups, the latter being submitted to the modified multiple platform paradigm for $96 \mathrm{~h}$. Subsets of eight rats in each group/experiment were submitted to the test of the tasks, either immediately or at different time intervals after SD. The results indicated that (a) pre- but not post-training SD impaired recall in the contextual and TFC; (b) this impairment was not state-dependent; and (c) in the contextual fear conditioning (CFC), pre-test SD prevented extinction of the learned task. Overall, these results suggest that SD interferes with acquisition, recall and extinction, but not necessarily with consolidation of emotional memory.

\section{Keywords: learning, memory, sleep deprivation, acquisition, consolidation, retrieval, extinction}

\section{INTRODUCTION}

A reciprocal influence between sleep and memory has been suggested, insofar as consolidation of newly acquired information is facilitated by previous sleep periods, whereas acquisition of new information alters sleep pattern (Maquet, 2001; Stickgold et al., 2001; Smith et al., 2004; Walker and Stickgold, 2004, 2005). Additional evidence of this relationship has been given by human and animal studies demonstrating that training in different memory tasks increases sleep time (Lucero, 1970; Smith et al., 1980; Smith and Lapp, 1986; Portell-Cortés et al., 1989; Smith and Rose, 1997) and that either sleep- (SD) or REM sleep deprivation (REMSD) before training impairs the performance of animals in numerous hippocampal-dependent tasks, such as inhibitory avoidance (Stern, 1971; Bueno et al., 1994, 2000; Gruart-Masso et al., 1995; Moreira et al., 2003; Dubiela et al., 2005), multiple trial inhibitory avoidance (Moreira et al., 2010; Ota et al., 2013), Morris water maze (Smith and Rose, 1996; Guan et al., 2004) and fear conditioning (Hicks et al.,
1988; Dametto et al., 2002; McDermott et al., 2003; Tiba et al., 2008). However, results from studies using pre-training protracted REMSD protocols are difficult to interpret because the animals are under an altered sleep-waking pattern both before (sleep-deprived) and after training (when sleep rebound is taking place) thus, precluding conclusions as to whether this manipulation affects acquisition and/or consolidation of the information in these memory tasks. In addition, REMSD-induced memory impairment may be due to its effect on attention, since this is disrupted by 72 or $96 \mathrm{~h}$ of REMSD and recovered by $24 \mathrm{~h}$ of sleep rebound (Godoi et al., 2005), suggesting that acquisition may be compromised. Indeed, sleep-deprived rats require more trainings in the multiple inhibitory avoidance task than control rats, and even so, their performance is poorer when tested $24 \mathrm{~h}$ later (Moreira et al., 2010; Ota et al., 2013). Finally, the possibility exists that impairment of performance in the abovementioned studies is due to a phenomenon known as state-dependent learning. This phenomenon implies in learning a particular response under a 
given stimulus-situation that involves a conditioning aspect; in test situations, when the training stimulus is replaced by another one, there is no transfer of the learned response (Overton, 1974). Therefore, in state-dependent learning a particular response is displayed if, and only if, there exists a similarity of the animal's inner state between the training and testing conditions (Colpaert et al., 1976).

Studies employing passive avoidance task show that $24 \mathrm{~h}$ of sleep recovery are sufficient to restore the performance of $96 \mathrm{~h}$ REM sleep-deprived rats (Dubiela et al., 2005, 2010), suggesting that SD-induced performance deficit may not be due to impairment of consolidation, but rather to some other unspecific effect, such as altered motor performance. In fact, REMSD inducedincreased locomotor activity in rats has been reported by some authors in rats (Suchecki et al., 2002; Tartar et al., 2009) and mice (Armani et al., 2012), but not by others (Dubiela et al., 2011); in the case of passive avoidance task, altered motor activity could be responsible for the short- ( $2 \mathrm{~h}$ post-training), but not for the long-term (24 h post-training) retention deficit (Dubiela et al., 2005), indicating, once again, that acquisition is impaired by REMSD.

One strategy to circumvent the impact of SD on acquisition is to sleep-deprive animals after training, so acquisition is guaranteed. The studies that employed this approach resulted in controversial results, with impairing (Graves et al., 2003; Palchykova et al., 2006), as well as improving effects (Gisquet-Verrier and Smith, 1989; Smith, 1996; Smith et al., 1998). This discrepancy may be attributed to the length $(5 \mathrm{~h} / 6 \mathrm{~h} \times 24 \mathrm{~h})$ and to the type of SD (total $\times$ REMSD). Moreover, the interval between SD and testing is an important methodological issue that should be considered when evaluating its effects in different memory stages. For instance, Silva et al. (2004) showed that 72 h of REMSD after training did not induce memory impairment in passive avoidance test or in the plus maze discriminative test when testing took place immediately after the end of SD, but when animals were re-tested 1 week later, they displayed impaired performance. Whether this impairment represents a facilitated extinction of the fear memory was not explored in this study, but there is evidence that $6 \mathrm{~h}$ of REMSD retard extinction of cued fear memory (Silvestri and Root, 2008).

Given the controversy regarding the effects of protracted SD on mnemonic process, the aim of the present study was to investigate the consequences of this manipulation, applied in different moments of memory formation in two related tasks that recruit distinct neural circuits, contextual fear conditioning (CFC), a hippocampal-dependent task, and tone fear conditioning (TFC), a hippocampal-independent task. Moreover, we also tested whether the performance impairment observed in these tasks could be explained by the state-dependent learning.

\section{METHODS}

\section{ANIMALS}

Male Wistar rats, aged 3 months, were obtained from the breeding colony of the Department of Psychobiology-UNIFESP. The animals were kept in groups of four in plastic cages $(40 \times 30 \times$ $17 \mathrm{~cm}$ ), filled with sawdust bedding, in a room under controlled temperature $\left(23^{\circ} \mathrm{C} \pm 2^{\circ} \mathrm{C}\right)$ and light/ dark cycle (lights on from
07:00 h to 19:00 h), with food and water provided ad libitum. The experimental protocols were approved by the Animal Care and Use Committee of UNIFESP (CEP \# 0943/06) and were in accordance with National Institutes of Health guidelines on animal care.

\section{SD PROCEDURE}

Rats were sleep deprived by the modified multiple platform method for $96 \mathrm{~h}$. SD was conducted by placing eight rats in a large water tank $(145 \times 30 \times 41 \mathrm{~cm})$ containing 12 narrow platforms $(6.5 \mathrm{~cm}$ in diameter). Previous studies from our group show that the platform method (either single or multiple) completely abolishes REM sleep but also decreases slow-wave sleep by approximately 35-40\% (Machado et al., 2004, 2008, 2013). The presence of cage-mates and multiple platforms prevent social isolation and movement restriction associated with earlier versions of SD (Suchecki and Tufik, 2000). Animals in the control (CTL) group remained in their home cages (HCs) in the same room where the SD procedure took place and were placed daily in the water tank for $1 \mathrm{~h}$, between 12:00 h and 13:00 h. This procedure was implemented so both sleep deprived and CTL groups would be removed from the same environment (water tank) before being submitted to the different protocols.

\section{APPARATUS}

The fear conditioning task apparatus consisted of an acrylic box, measuring $21 \times 26 \times 27.5 \mathrm{~cm}$. The apparatus had black walls and transparent acrylic top. The floor consisted of a metal grid $(0.4 \mathrm{~cm}$ diameter rods placed $1.2 \mathrm{~cm}$ apart) connected to a shock generator and control module (AVS-Projetos Especiais, São Paulo, Brazil), through which footshocks could be delivered.

The TFC test was carried out using a white cylindrical test chamber $(35 \mathrm{~cm}$ in diameter $\times 60 \mathrm{~cm}$ high), with a transparent lid containing small holes. The apparatuses were placed in different rooms, being connected to a buzzer located outside the conditioning apparatus and outside the cylindrical chamber $(60 \mathrm{~dB})$ activated by a manual switch and used as the conditioned stimulus (CS).

\section{BEHAVIORAL PROCEDURE}

Contextual fear conditioning (CFC): Training was performed in one session, in which the animals were individually placed in the conditioning chamber where they remained for $2 \mathrm{~min}$. The behavior of each animal was recorded continuously by measuring the time in freezing behavior (defined as complete immobility and absence of vibrissae movements) minute by minute. After this period, rats received five footshocks $(0.7 \mathrm{~mA}, 1 \mathrm{~s}$ long $)$ at $30 \mathrm{~s}$ intervals and were removed from the apparatus $1 \mathrm{~min}$ after the last footshock. CFC tests were performed after different post-training periods, depending on the experiment protocol. During the test, rats were placed in the same training context, and no footshock was delivered. The time in freezing was again recorded minute by minute for $5 \mathrm{~min}$. Freezing time/min ratio was taken as the performance of contextual conditioning. 
Tone fear conditioning: Training was performed in one session, identical to CFC, but in this case, a sound stimulus (60 $\mathrm{dB}-5 \mathrm{~s}$ long) was delivered, finishing concomitantly with a 1 s long footshock $(0.7 \mathrm{~mA})$. Five tone-footshock pairings were delivered, $30 \mathrm{~s}$ apart. During the TFC test (which also took place after different time intervals after training), rats were placed in the cylindrical chamber (new context, different room) and after 2 min the sound was presented 5 times, in the same schedule as during training, without release of any footshock and the freezing time /min was taken as the conditioning measure.

\section{EXPERIMENTS 1, 2 AND 3}

Different groups of animals ( $N=8 /$ group/task/experiment) were trained in the CFC or in the TFC and then distributed into either $96 \mathrm{~h}$ of SD or CTL groups. In Experiment 1A, the groups were tested in the respective tasks immediately after the end of the SD period (or at the equivalent time for the CTL group) (Figure 1, upper panel). In Experiment 2, rats in the SD group were allowed to sleep for $24 \mathrm{~h}$ before the test, in order to examine whether the manipulation interfered with memory consolidation or retrieval (Figure 2, upper panel). In Experiment 3, sleep-deprived animals were allowed to sleep for $96 \mathrm{~h}$ before being tested, a period sufficient to guarantee return to normal sleep pattern (Machado et al., 2004). The experimental procedure is shown in Figure 3 (upper panel).

\section{EXPERIMENT 4}

To discard the possibility that the results of Experiments 1A, B and $\mathrm{C}$ were due to a state-dependent learning, different sets of animals were distributed in one of four groups: G I-remained in the HC for the whole experimental period; G II-Submitted to SD only after training; G III-submitted to SD only before training; G IV-submitted to SD both before and after training, thus being sleep-deprived during acquisition and retrieval of the learned material. Consequently, both G I and G IV were in the same state during both training and test sessions, and if indeed there was a state-dependent learning then these groups should not exhibit memory impairment. On the other hand, if memory impairment were observed only for pre-training or pretest sessions as shown previously (both in the present study and in the literature), then only $\mathrm{G}$ I should perform adequately during the test (the experimental procedure is shown in Figure 4, upper panel).

\section{EXPERIMENT 5}

The previous results suggested that SD impaired recall of context fear conditioning. However, it is not possible to completely rule out the possibility that SD impaired the ability of animals to freeze (an effect on motor activity). To rule out this possibility, we exposed trained rats to three tests in CFC and TFC, separated by 8 days from each other. Different groups of animals were trained and then submitted to $96 \mathrm{~h}$ of SD at different moments: before test 1 (G2), after test 1 (G3) or in the absence of the first re-exposure

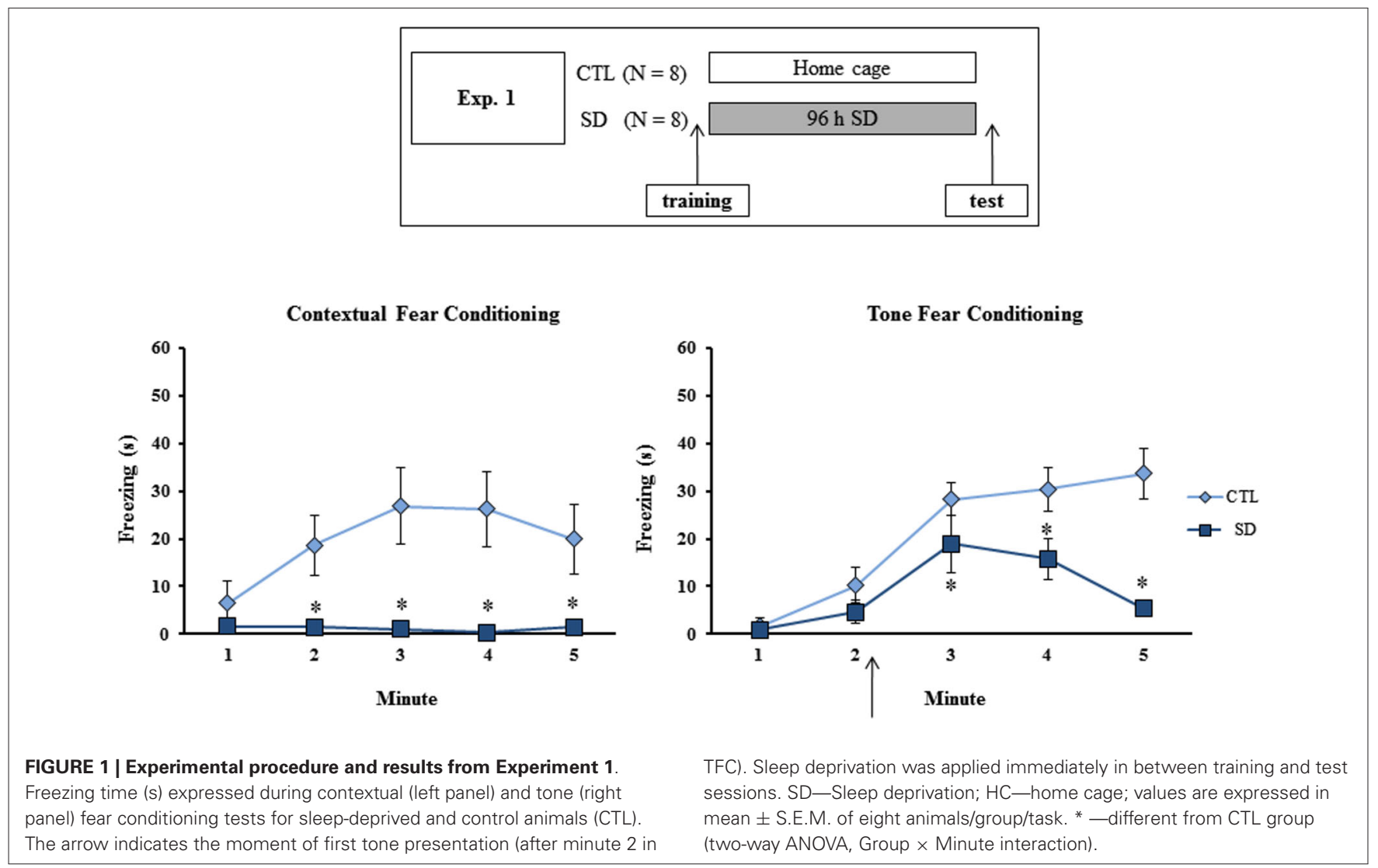




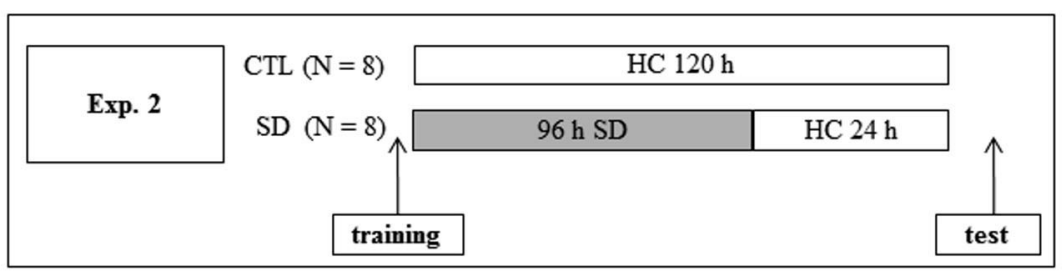

Contextual Fear Conditioning

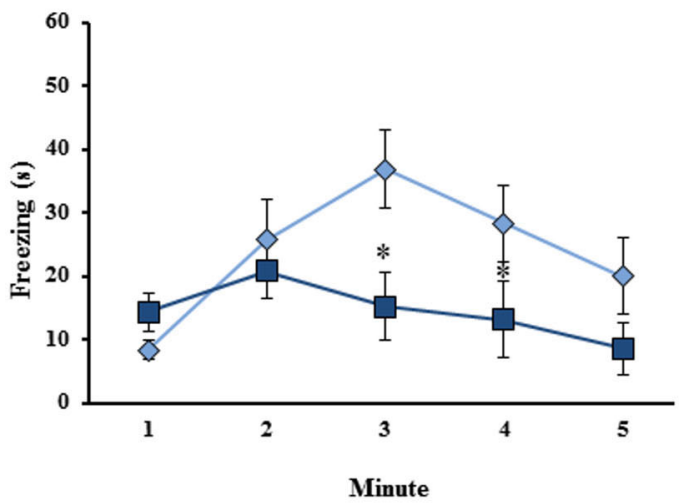

FIGURE 2 | Experimental procedure and results from Experiment 2. Freezing time (s) expressed during contextual (left panel) and tone (right panel) fear conditioning tests for sleep-deprived and control animals (CTL). The arrow indicates the moment of first tone presentation (after minute 2 in TFC). Sleep deprivation was applied

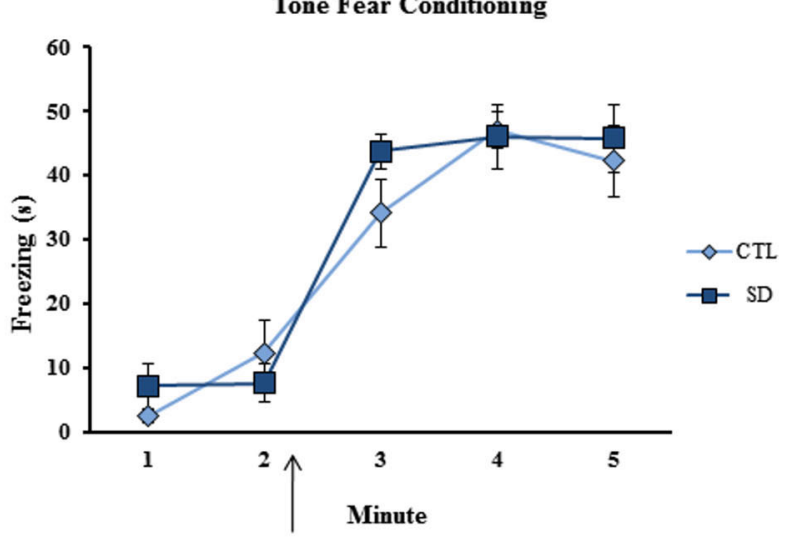

after training session and test session occurred after $24 \mathrm{~h}$ in which the animals remained in their home cages. SD-Sleep deprivation; $\mathrm{HC}$-home cage; values are expressed in mean \pm S.E.M. of eight animals/group/task. * - different from CTL group (two-way ANOVA, Group $\times$ Minute interaction). to the conditioning chamber (G4). CTL group (G1) remained in the $\mathrm{HC}$ during the entire experimental protocol as can be seen in Figure 5 (upper panel).

\section{STATISTICAL ANALYSIS}

Data from Experiments 1A, B and C, were analyzed by a twoway ANOVA for repeated measures, with Group $(\mathrm{SD} \times \mathrm{CTL})$ and Minute (repeated measure: 1-5) as main factors. For Experiment 2, a two-way ANOVA for dependent variables was employed with Group (G I-G IV) and Minute (repeated measure: $1-5$ ) as main factors. Analysis of Experiment 3 used a one-way ANOVA for each session (training, test 1 , test 2 and test 3 ), considering four groups (G1-G4; except for test 1, in which G4 was not tested). When necessary, a post hoc analysis was performed by the NewmanKeuls test, with a $P$-value $\leq 0.05$ being considered statistically significant.

\section{RESULTS}

\section{EXPERIMENT 1: EFFECTS OF SD IMMEDIATELY BEFORE TESTING IN FEAR CONDITIONING TASKS}

During training session, no difference in freezing time between the groups was observed on either task (data not shown). During CFC test, main effects of Group $\left(F_{(1,14)}=9.66 ; p<0.01\right)$ and Minute $\left(F_{(4,56)}=3.82 ; p<0.01\right)$ and an interaction between these factors $\left(F_{(4,56)}=4.67 ; p<0.005\right)$ were revealed, where SD group exhibited less freezing time than CTL animals at 2, 3, 4 and $5 \mathrm{~min}$ (Figure 1, left panel).
In the TFC task, main effects of Group $\left(F_{(1,14)}=9.37 ; p<0.01\right)$ and Minute $\left(F_{(4,56)}=24.23 ; p<0.0001\right)$ and an interaction between these factors $\left(F_{(4,56)}=6.65 ; p<0.001\right)$ were also observed. Post-hoc analysis showed that SD rats displayed less freezing time than CTL rats after the tone, although the groups did not differ before presentation of the CS (Figure 1, right panel).

\section{EXPERIMENT 2: EFFECTS OF 24 H SLEEP RECOVERY ON TESTING OF FEAR CONDITIONING TASKS}

No difference in freezing time was found during training of either task, or for the test in TFC. However a Group $\times$ Minute interaction was found $\left(F_{(4,52)}=4.56 ; p<0.003\right)$ for CFC and the post hoc analysis revealed that SD animals displayed less freezing during minutes 3 and 4 than CTL rats (Figure 2).

\section{EXPERIMENT 3: EFFECTS OF 96 H SLEEP RECOVERY ON TESTING OF FEAR CONDITIONING TASKS}

No difference in freezing time was found during training or test of either task. In TFC, a Minute effect was found $\left(F_{(4,52)}=98.75\right.$; $p<0.001$ ), and freezing behavior was equally increased after tone presentation in both CTL and SD groups (Figure 3, left panel).

\section{EXPERIMENT 4: STATE-DEPENDENT EFFECT OF SD ON FEAR CONDITIONING TASKS}

Analysis of CFC revealed a Group effect for training session $\left(F_{(3,28)}=7.84 ; p<0.0001\right)$, as well as a Minute effect $\left(F_{(2,56)}=136.02 ; p<0.001\right)$ and a Group $\times$ Minute interaction 


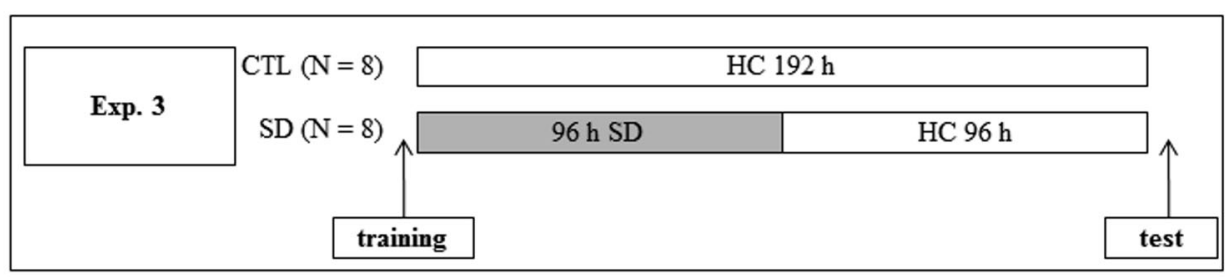

Contextual Fear Conditioning

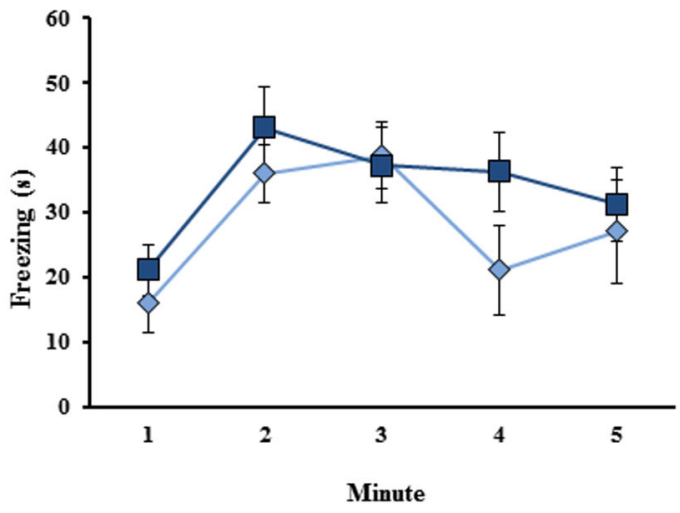

FIGURE 3 | Experimental procedure and results from Experiment 3. Freezing time (s) expressed during contextual (left panel) and tone (right panel) fear conditioning tests for sleep-deprived and control animals (CTL). The arrow indicates the moment of first tone presentation (after minute 2 in TFC). Sleep deprivation was applied

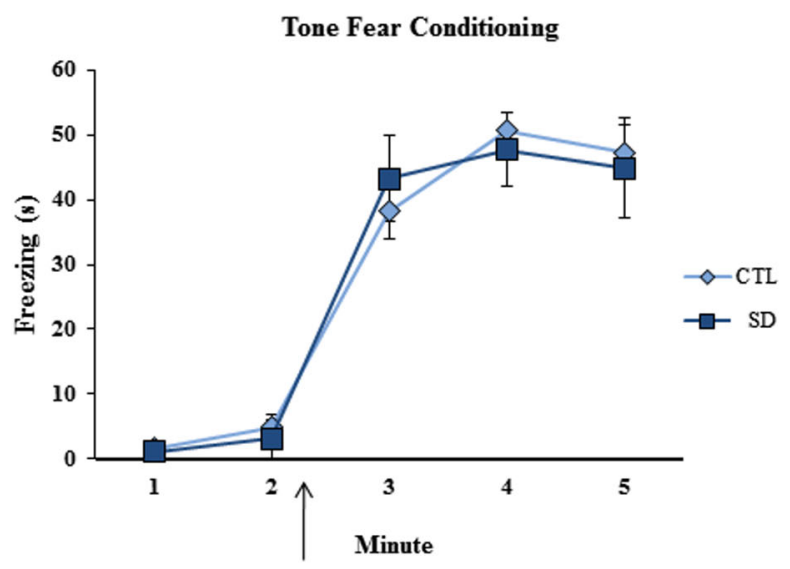

after training session and test session occurred after $96 \mathrm{~h}$ in which the animals remained in their home cages. SD-sleep deprivation; $\mathrm{HC}$-home cage; values are expressed in mean \pm S.E.M. of eight animals/group/task. * - different from CTL group (two-way ANOVA, Group $\times$ Minute interaction).
$\left(F_{(6,56)}=11.96 ; p<0.001\right)$. The Newman-Keuls test showed that freezing time was lower for GI (CTL group) and GII (rats sleep-deprived immediately before testing) than for GIII (rats sleep-deprived immediately before training) and GIV (rats sleep-deprived immediately before training and before testing), which were not different from each other (data not shown). Analysis of the animals' performance during the test revealed main effects of Group $\left(F_{(3,28)}=51.64 ; p<0.001\right)$ and Minute $\left(F_{(4,112)}=6,79 ; p<0,0001\right)$ and an interaction between these factors $\left(F_{(12,112)}=7.52 ; p<0.001\right)$. Newman-Keuls test showed that GI was different from all other groups, which did not differ from each other (Figure 4, left panel).

For TFC training, there was no Group effect or Group $\times$ Minute interaction, but only a Minute effect $\left(F_{(2,48)}=89.69\right.$; $p<0.001)$. During test, two-way ANOVA revealed main effects of $\operatorname{Group}\left(F_{(3,24)}=35.21 ; p<0.001\right)$ and Minute $\left(F_{(4,96)}=42.96\right.$; $p<0.0001)$ and a Group $\times$ Minute interaction $\left(F_{(12,96)}=7.03\right.$; $p<0.001$ ); GI was different from all other groups, which were not different from each other (Figure 4, right panel).

\section{EXPERIMENT 5: EFFECT OF SD ON MULTIPLE TESTS IN FEAR CONDITIONING TASKS}

No differences were observed during training sessions in either task. In regard to the animals' performance in the CFC, there was a main effect of Group on test $1\left(F_{(2,20)}=20.66 ; p<0.00005\right)$, test $2\left(F_{(3,27)}=4.76 ; p<0.01\right)$ and test $3\left(F_{(3,27)}=6.32 ; p<0.005\right)$.
Post hoc analysis showed that on test 1, G2 was different from G1 (CTL group) and G3 (rats sleep-deprived immediately before test $1 ; p<0.0005$ ); on test 2, G2 and G4 (rats were sleep-deprived as G2, but were submitted to test 1) were different from G1, but not from G3 and on test 3, again, only G2 was different from all other groups ( $p<0.01$ ) (Figure 5, left panel).

For TFC, there was no difference among groups during training, test 2 or test 3. For test 1, a Group effect was found $\left(F_{(2,21)}=7.90 ; p<0.005\right)$ and the post hoc analysis revealed that only G2 was different from G1 and G3 ( $p<0.01$; Figure 5, right panel).

\section{DISCUSSION}

The results of the present study showed that SD affected memory acquisition and retrieval, but not consolidation; this effect was not induced by a state-dependent learning and exposure of animals to SD only immediately before the first retrieval session impaired the extinction of a hippocampus-dependent task.

In Experiments 1, 2 and 3, we investigated whether SDinduced impairment of recall in aversively motivated tasks, in the present case, CFC and TFC, resulted from interference with memory consolidation and/or recall per se. The results of these experiments strongly suggest that SD impairs recall, but not consolidation, because the performance of sleep-deprived rats in the TFC task was restored to control values after $24 \mathrm{~h}$ of 


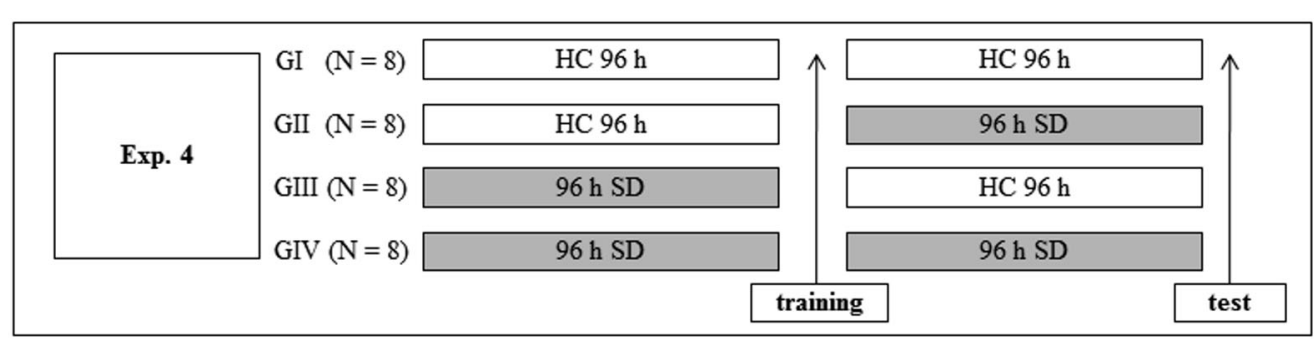

Contextual Fear Conditioning

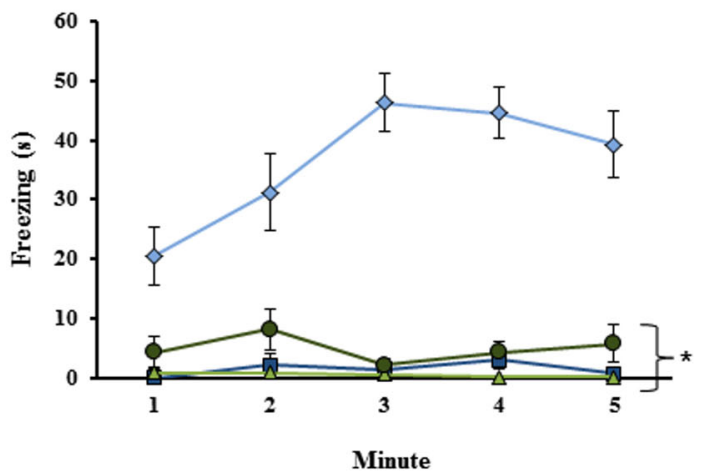

FIGURE 4 | Experimental procedure and results from Experiment 4. Freezing time (s) expressed during contextual (left panel) and tone (right panel) fear conditioning test for sleep-deprived and control animals (CTL). The arrow indicates the moment of first tone presentation (after minute 2 in TFC). Sleep deprivation was applied at different moments for each group. To

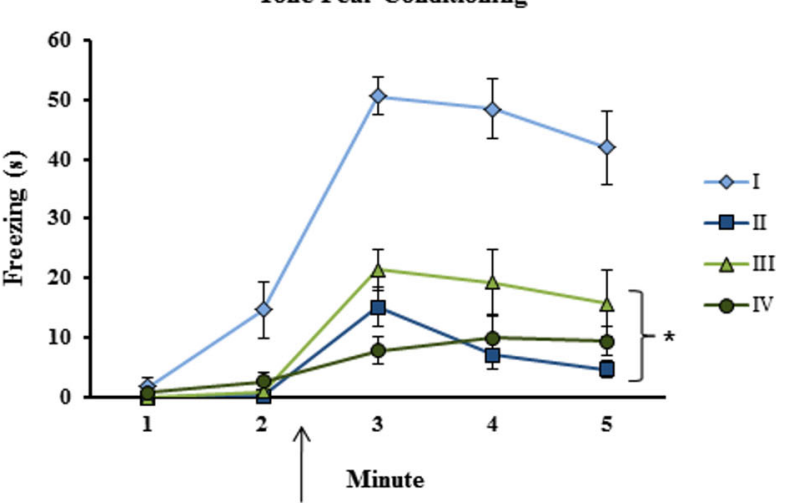

confirm state dependent learning, groups that were in the same state during both training and test session (G I and G IV) should perform better than the others (G II and G III). SD-sleep deprivation; HC-home cage; values are expressed in mean \pm S.E.M. of eight animals/group/task. ${ }^{*}$ —all groups were different from G I (CTL group - two-way ANOVA, Group effect).

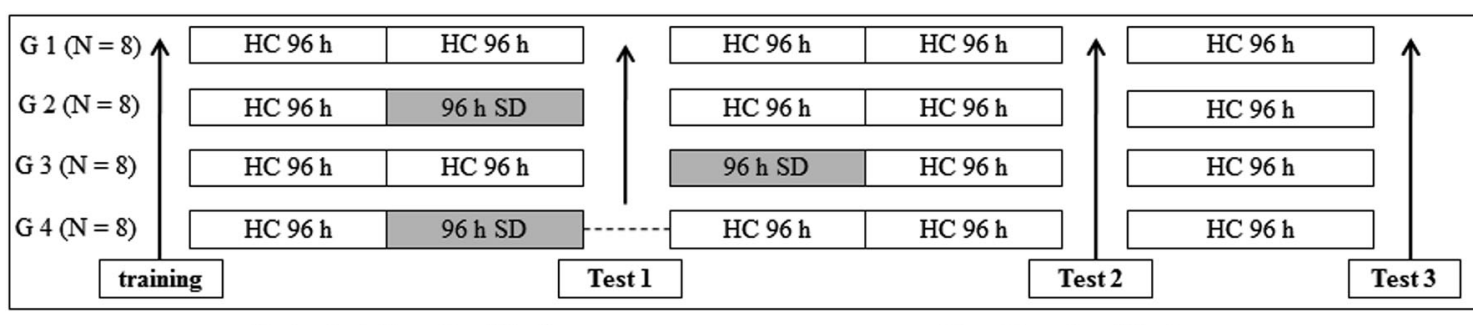

Contextual Fear Conditioning

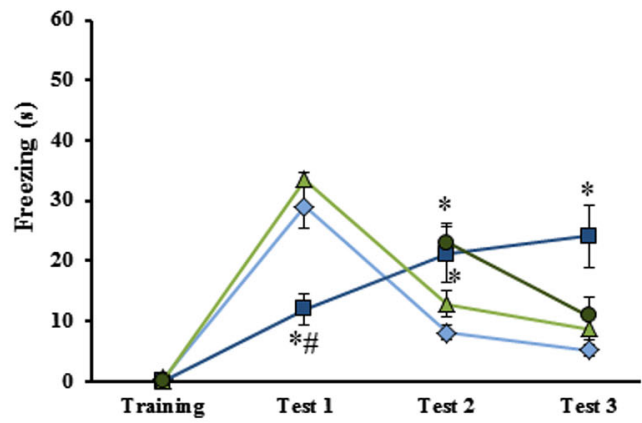

FIGURE 5 | Experimental procedure and results from Experiment 5 Freezing time (s) expressed during contextual (left panel) and tone (right panel) fear conditioning test for sleep-deprived and control animals (CTL). Sleep deprivation was applied at different moments for each group: before

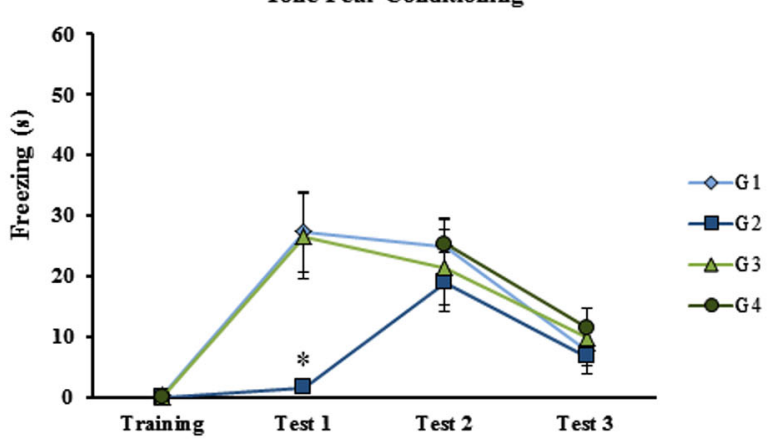

test 1 for G2; after test 2 for G3 and for G4, sleep deprived animals were not submitted to test 1. SD—sleep deprivation; HC-home cage; values are expressed in mean \pm S.E.M. of eight animals/group. * — different from $\mathrm{G} 1$ (CTL group-one-way ANOVA, Group effect). sleep recovery, whereas in the CFC, the same effect was obtained between 24 and $96 \mathrm{~h}$ of sleep recovery. Interestingly, previous studies have shown that short periods of SD immediately (Graves et al., 2003; Palchykova et al., 2006) or 1 h (Prince et al., 2014) 
after training in hippocampus-dependent tasks impair memory consolidation and, consequently, recall. One possible explanation for the lack of SD effect on consolidation, may reside in the amount of associations made between footshock and either context or tone; in Graves et al. (2003), mice received one footshock of $1.5 \mathrm{~mA}$, whereas in the present one, rats received five footshocks of $0.8 \mathrm{~mA}$, and there is a possibility that memory consolidation begins already after the first pairing. Nonetheless, this possibility appears to be true for CFC, whereas data on the effects of SD on TFC are controversial, with studies showing this task to be resistant to the disrupting effects of REMSD (Bueno et al., 1994; Ruskin et al., 2004; Ruskin and Lahoste, 2009) or SD (Graves et al., 2003) and others showing that it is also affected by SD (Dametto et al., 2002; Kumar and Jha, 2012). These findings may explain why a shorter period of sleep recovery was already enough to restore the animals' performance in TFC, whereas in CFC, a period between $24 \mathrm{~h}$ and $96 \mathrm{~h}$ was required for animals to express their recall-related behavior.

It has been shown previously that $96 \mathrm{~h}$ of SD by the platform method leads to intense sleep rebound in the $24 \mathrm{~h}$ following the deprivation period (Machado et al., 2004, 2008, 2013) resulting in disorganized sleep pattern characterized by excessive compensation of REM sleep and reduced non-REM sleep. Impairment of recall could also be attributed to this altered sleep architecture as recent studies have shown that non-REM sleep is also important for memory performance in emotional tasks (Giuditta et al., 1995; Fogel and Smith, 2006; Hellman and Abel, 2007). By allowing animals to sleep for $96 \mathrm{~h}$ between SD and testing, sleep pattern as well as the performance of sleep-deprived rats returned to normal on both tasks, being identical to that of control rats, thus evidencing that SD affects retrieval, but not memory consolidation. In addition, these data indicate a dissociative effect of SD on CFC and TFC, reinforcing the proposition that the impact of SD is not unspecific (as, for instance, on motor activity) since both tasks require the same response: freezing behavior.

The results of Experiment 4 indicated that memory impairment in both CFC and TFC was not due to a state-dependent learning. If it were, then animals that were sleep deprived before training and before testing should display a performance similar to that of control animals. However, the performance in both tasks was impaired in animals sleep-deprived either before training and/or testing. These results are in contrast with a previous one (Patti et al., 2010), that investigated the state-dependent effects of $72 \mathrm{~h}$ of REMSD in mice. Possible sources of controversy may be the use of different species, length of SD, but most of all, differences in the experimental protocol, for in Patti et al.'s study, testing was performed 7 days after training, a time sufficient for the animals to sleep recover and, according to the present results, to restore the performance in hippocampusdependent tasks. In the present study, there was no interval between training and testing other than the period involving SD.

Just as REMSD applied before training may impair performance by altering attention state during acquisition so can the same phenomenon be true for retrieval. As, to the best of our knowledge, no study had ever been performed to evaluate the effects of SD on attentional process during retrieval, this possibility cannot be ruled out. Thus, even if acquisition and consolidation phases were guaranteed (Experiments $1 \mathrm{~B}$ and $\mathrm{C}$ ), pre-test SD might still affect unspecific aspects of performance rather than impairing retrieval per se. Importantly, during retrieval, memories become labile and may undergo two different processes: one that weakens old memories as a result of extinction and another that strengthens the memory as a result of additional consolidation, a process known as reconsolidation. These processes share common mechanisms but also recruit distinct circuits (for review, see (Alberini, 2005). Carrying out subsequent tests, in which the animals would be no longer sleep-deprived, could be a useful strategy to assure that normal performance was restored and to evaluate specific aspects of memory retrieval. Thus, in Experiment 5, animals were sleep-deprived either immediately before or after the first test, in order to investigate whether the manipulation would interfere with post-learning mechanisms. The results confirmed the impairing effects of SD before testing, but fear memory was expressed again in a subsequent test of both CFC and TFC, when rats had already recovered from SD (test 2). As expected, all groups, except from that sleep-deprived before test 1 of CFC exhibited low levels of freezing behavior upon repeated exposures to the CS, indicating that SD before recall of hippocampus-dependent fear memory had a long-term effect on memory processing. This result is unlikely to reflect SDinduced fear memory erasure, since SD when not followed by testing (G4 in Experiment 5) did not affect memory extinction. Interestingly, although freezing behavior was not expressed by rats of G2, their memory became labile, allowing some kind of alteration to take place leading to persistence of the fear memory. Several possibilities can be offered to explain the effect of SD conducted just prior to the first test. The first one refers to the acute effect on memory retrieval: although this may be the case, it is not possible to dissociate this effect from the confounding effect of SD on motor performance or emotionality. Usually a retrieval deficit can be inferred when the manipulation affects only one task, but in the present experiments both tasks were affected. Nonetheless, the long lasting effect of SD selectively observed in CFC cannot be attributed to an acute influence on recall or motor performance. The second one refers to the impairing effect of SD on extinction or, alternatively, on reconsolidation, or both. As control rats extinguished the conditioned response it is reasonable to assume that sleep manipulation interfered with this phenomenon. Because stressful situations disrupt reconsolidation (Cai et al., 2006) and SD induces sustained increase of corticosterone plasma levels (Galvão et al., 2009), it is unlikely that SD would produce a positive effect on reconsolidation of fear memories.

Pre-test SD affected memory extinction only for CFC, but not for TFC task, a result that is in contrast with previous work reporting that REMSD affects only TFC but not CFC (Silvestri, 2005; $\mathrm{Fu}$ et al., 2007). Some methodological differences may explain this contradiction. In Silvestri's work, rats were submitted to $6 \mathrm{~h}$ of REMSD immediately after training, whereas testing/extinction training occurred $48 \mathrm{~h}$ later. Sleep-deprived animals did not extinguish fear behavior in TFC, but freezing was reduced in 
CFC. In a subsequent test, no difference was found in TFC, and all animals expressed similar levels of fear behavior, whereas there was a tendency to increase freezing in REMSD animals in CFC. This later result would agree with the present data, in which SD animals presented impaired extinction in subsequent tests only in CFC. Importantly, in Silvestri's work training/testing and extinction for TFC were carried out in the same chamber, while in the present study TFC test and extinction was performed in a chamber different from the training one. The importance of context on modulation of extinction performance has been reviewed elsewhere (Bouton et al., 2006). Although Fu et al.'s work took this aspect into consideration, the same result was reported, i.e., impaired extinction in the TFC but not in the CFC after SD. There are still some methodological differences among these studies that could account for the distinct outcomes reported, including habituation prior to training (used in both studies20 and 30 min respectively-but not in the present one), that could facilitate contextual extinction, masking possible effects of REMSD on extinction of this task, different lengths of SD (6 h after training in both studies), interval between training and testing (shorter periods in the previous studies and $96 \mathrm{~h}$ in the present one) and different methods of SD. Naturally, it is only possible to establish which factor(s) could influence the outcomes with certainty, by performing a study that compares all these variables.

Nevertheless, despite the inconsistencies between the abovementioned studies and the present one, the outcomes seem to convey the message that sleep loss can be detrimental for extinguishing aversive memories. This conclusion is in accordance with reports in human beings who had REMS periods during a 90-min nap and displayed less reaction to fearful facial expressions and more positive reactions to happy faces (Gujar et al., 2011) and a report that individuals who were involved in automobile accidents were more likely to develop depression and posttraumatic stress disorder (PTSD) when experienced sleep disturbances on the 2 weeks prior to the traumatic event (Bryant et al., 2010); conversely, individuals who displayed consolidated REM sleep in the aftermath of a traumatic situation are less likely to develop PTSD (Mellman et al., 2007). Collectively, these findings agrees with the proposition that REM sleep facilitates reframing of negative experiences and restores emotional reactivity (Walker and Van Der Helm, 2009). Therefore, experimental protocols such as those used in Silvestri (2005), Fu et al. (2007) and in Experiment 3 are promising tools to study the neural circuits involved in the relationship between REM sleep and extinction of fear memory.

In conclusion, $96 \mathrm{~h}$ of SD immediately after training of aversive memory tasks impaired the recall of fear memory both in context and TFC and this effect was reversed after a period of sleep recovery, but did not seem to result from a state-dependent learning process. However, when SD took place immediately before testing, there was an impairment of extinction of the aversive memory only in CFC.

\section{ACKNOWLEDGMENTS}

The authors are in debt to Marcos Vinicius Bunscheit for assistance in the behavioral testing and animal care. This work was supported by Associação Fundo de Incentivo à Pesquisa (AFIP). Vanessa Contatto Rossi and Karin Di Monteiro Moreira were recipients of Undergraduate and $\mathrm{PhD}$ fellowships from FAPESP, respectively. Paula Ayako Tiba was the recipient of a post-doc fellowship from Conselho Nacional de Desenvolvimento Científico e Tecnológico (CNPq); Deborah Suchecki and Maria Gabriela Menezes Oliveira are the recipients of research fellowships from CNPq. Payment for this article was made possible by a grant from FAPESP (grant \#: 2012/ 23488-6).

\section{REFERENCES}

Alberini, C. M. (2005). Mechanisms of memory stabilization: are consolidation and reconsolidation similar or distinct processes? Trends Neurosci. 28, 51-56. doi: 10. 1016/j.tins.2004.11.001

Armani, F., Andersen, M. L., Andreatini, R., Frussa-Filho, R., Tufik, S., and Galduróz, J. C. (2012). Successful combined therapy with tamoxifen and lithium in a paradoxical sleep deprivation-induced mania model. CNS Neurosci. Ther. 18, 119-125. doi: 10.1111/j.1755-5949.2010.00224.x

Bouton, M. E., Westbrook, R. F., Corcoran, K. A., and Maren, S. (2006). Contextual and temporal modulation of extinction: behavioral and biological mechanisms. Biol. Psychiatry 60, 352-360. doi: 10.1016/j.biopsych.2005.12.015

Bryant, R. A., Creamer, M., O'donnell, M., Silove, D., and Mcfarlane, A. C. (2010). Sleep disturbance immediately prior to trauma predicts subsequent psychiatric disorder. Sleep 33, 69-74.

Bueno, O. F., Lobo, L. L., Oliveira, M. G., Gugliano, E. B., Pomarico, A. C., and Tufik, S. (1994). Dissociated paradoxical sleep deprivation effects on inhibitory avoidance and conditioned fear. Physiol. Behav. 56, 775-779. doi: 10.1016/00319384(94)90241-0

Bueno, O. F., Oliveira, G. M., Lobo, L. L., Morais, P. R., Melo, F. H., and Tufik, S. (2000). Cholinergic modulation of inhibitory avoidance impairment induced by paradoxical sleep deprivation. Prog. Neuropsychopharmacol. Biol. Psychiatry 24, 595-606. doi: 10.1016/s0278-5846(00)00095-6

Cai, W. H., Blundell, J., Han, J., Greene, R. W., and Powell, C. M. (2006). Postreactivation glucocorticoids impair recall of established fear memory. J. Neurosci. 26, 9560-9566. doi: 10.1523/jneurosci.2397-06.2006

Colpaert, F. C., Niemegeers, C. J., and Janssen, P. A. (1976). Theoretical and methodological considerations on drug discrimination learning. Psychopharmacologia 46, 169-177. doi: 10.1007/bf00421388

Dametto, M., Suchecki, D., Bueno, O. F., Moreira, K. M., Tufik, S., and Oliveira, M. G. (2002). Social stress does not interact with paradoxical sleep deprivationinduced memory impairment. Behav. Brain Res. 129, 171-178. doi: 10. 1016/s0166-4328(01)00345-x

Dubiela, F. P., Messias, M. F., Moreira, K. D., Zanlorenci, L. H., Grassl, C., Filho, R. F., et al. (2011). Reciprocal interactions between MK-801, sleep deprivation and recovery in modulating rat behaviour. Behav. Brain Res. 216, 180-185. doi: 10.1016/j.bbr.2010.07.031

Dubiela, F. P., Oliveira, M. G., Moreira, K. M., Nobrega, J. N., Tufik, S., and Hipólide, D. C. (2005). Learning deficits induced by sleep deprivation and recovery are not associated with altered $[(3) \mathrm{H}]$ muscimol and $[(3) \mathrm{H}]$ flunitrazepam binding. Brain Res. 1037, 157-163. doi: 10.1016/j.brainres.2005. 01.005

Dubiela, F. P., Oliveira, M. G., Moreira, K. M., Nobrega, J. N., Tufik, S., and Hipólide, D. C. (2010). Inverse benzodiazepine agonist beta-CCM does not reverse learning deficit induced by sleep deprivation. Neurosci. Lett. 469, 169173. doi: 10.1016/j.neulet.2009.11.070

Fogel, S. M., and Smith, C. T. (2006). Learning-dependent changes in sleep spindles and Stage 2 sleep. J. Sleep Res. 15, 250-255. doi: 10.1111/j.1365-2869.2006. 00522.x

Fu, J., Li, P., Ouyang, X., Gu, C., Song, Z., Gao, J., et al. (2007). Rapid eye movement sleep deprivation selectively impairs recall of fear extinction in hippocampus-independent tasks in rats. Neuroscience 144, 1186-1192. doi: 10. 1016/j.neuroscience.2006.10.050

Galvão, M. O. L., Sinigaglia-Coimbra, R., Kawakami, S. E., Tufik, S., and Suchecki, D. (2009). Paradoxical sleep deprivation activates hypothalamic nuclei that regulate food intake and stress response. Psychoneuroendocrinology 34, 11761183. doi: 10.1016/j.psyneuen.2009.03.003 
Gisquet-Verrier, P., and Smith, C. (1989). Avoidance performance in rat enhanced by postlearning paradoxical sleep deprivation. Behav. Neural Biol. 52, 152-169. doi: 10.1016/s0163-1047(89)90271-9

Giuditta, A., Ambrosini, M. V., Montagnese, P., Mandile, P., Cotugno, M., Grassi Zucconi, G., et al. (1995). The sequential hypothesis of the function of sleep. Behav. Brain Res. 69, 157-166. doi: 10.1016/0166-4328(95) 00012-i

Godoi, F. R., Oliveira, M. G., and Tufik, S. (2005). Effects of paradoxical sleep deprivation on the performance of rats in a model of visual attention. Behav. Brain Res. 165, 138-145. doi: 10.1016/j.bbr.2005.06.024

Graves, L. A., Heller, E. A., Pack, A. I., and Abel, T. (2003). Sleep deprivation selectively impairs memory consolidation for contextual fear conditioning. Learn. Mem. 10, 168-176. doi: 10.1101/lm.48803

Gruart-Masso, A., Nadal-Alemany, R., Coll-Andreu, M., Portell-Cortès, I., and Martí-Nicolovius, M. (1995). Effects of pretraining paradoxical sleep deprivation upon two-way active avoidance. Behav. Brain Res. 72, 181-183. doi: 10. 1016/0166-4328(96)00082-4

Guan, Z., Peng, X., and Fang, J. (2004). Sleep deprivation impairs spatial memory and decreases extracellular signal-regulated kinase phosphorylation in the hippocampus. Brain Res. 1018, 38-47. doi: 10.1016/j.brainres.2004.05.032

Gujar, N., Mcdonald, S. A., Nishida, M., and Walker, M. P. (2011). A role for REM sleep in recalibrating the sensitivity of the human brain to specific emotions. Cereb. Cortex 21, 115-123. doi: 10.1093/cercor/bhq064

Hellman, K., and Abel, T. (2007). Fear conditioning increases NREM sleep. Behav. Neurosci. 121, 310-323. doi: 10.1037/0735-7044.121.2.310

Hicks, R. A., Hicks, G. J., and Reyes, J. R. (1988). REM-Sleep deprivation and condiitoned fear in rats. Bull. Psychon. Soc. 26, 59-60. doi: 10.3758/bf033 34861

Kumar, T., and Jha, S. K. (2012). Sleep deprivation impairs consolidation of cued fear memory in rats. PLoS One 7:e47042. doi: 10.1371/journal.pone.0047042

Lucero, M. A. (1970). Lengthening of REM sleep duration consecutive to learning in the rat. Brain Res. 20, 319-322. doi: 10.1016/0006-8993(70) 90299-4

Machado, R. B., Hipólide, D. C., Benedito-Silva, A. A., and Tufik, S. (2004). Sleep deprivation induced by the modified multiple platform technique: quantification of sleep loss and recovery. Brain Res. 1004, 45-51. doi: 10.1016/j.brainres. 2004.01.019

Machado, R. B., Tufik, S., and Suchecki, D. (2008). Chronic stress during paradoxical sleep deprivation increases paradoxical sleep rebound: association with prolactin plasma levels and brain serotonin content. Psychoneuroendocrinology 33, 1211-1224. doi: 10.1016/j.psyneuen.2008.06.007

Machado, R. B., Tufik, S., and Suchecki, D. (2013). Role of corticosterone on sleep homeostasis induced by REM sleep deprivation in rats. PLoS One 8:e63520. doi: 10.1371/journal.pone.0063520

Maquet, P. (2001). The role of sleep in learning and memory. Science 294, 1048 1052. doi: 10.1126/science.1062856

McDermott, C. M., Lahoste, G. J., Chen, C., Musto, A., Bazan, N. G., and Magee, J. C. (2003). Sleep deprivation causes behavioral, synaptic and membrane excitability alterations in hippocampal neurons. J. Neurosci. 23, 9687-9695.

Mellman, T. A., Pigeon, W. R., Nowell, P. D., and Nolan, B. (2007). Relationships between REM sleep findings and PTSD symptoms during the early aftermath of trauma. J. Trauma. Stress 20, 893-901. doi: 10.1002/jts.20246

Moreira, K. M., Ferreira, T. L., Hipolide, D. C., Fornari, R. V., Tufik, S., and Oliveira, M. G. (2010). Modafinil prevents inhibitory avoidance memory deficit induced by sleep deprivation in rats. Sleep 33, 990-993.

Moreira, K. M., Hipólide, D. C., Nobrega, J. N., Bueno, O. F., Tufik, S., and Oliveira, M. G. (2003). Deficits in avoidance responding after paradoxical sleep deprivation are not associated with altered $[3 \mathrm{H}]$ pirenzepine binding to $\mathrm{M} 1$ muscarinic receptors in rat brain. Brain Res. 977, 31-37. doi: 10.1016/s00068993(03)02688-X

Ota, S. M., Moreira, K. D., Suchecki, D., Oliveira, M. G. M., and Tiba, P. A. (2013). Lithium prevents REM sleep deprivation-induced impairments on memory consolidation. Sleep 36, 1677-1684. doi: 10.5665/sleep.3126

Overton, D. A. (1974). Experimental methods for the study of state-dependent learning. Fed. Proc. 33, 1800-1813.

Palchykova, S., Winsky-Sommerer, R., Meerlo, P., Dürr, R., and Tobler, I. (2006). Sleep deprivation impairs object recognition in mice. Neurobiol. Learn. Mem. 85, 263-271. doi: 10.1016/j.nlm.2005.11.005
Patti, C. L., Zanin, K. A., Sanday, L., Kameda, S. R., Fernandes-Santos, L., Fernandes, H. A., et al. (2010). Effects of sleep deprivation on memory in mice: role of state-dependent learning. Sleep 33, 1669-1679.

Portell-Cortés, I., Martí-Nicolovius, M., Segura-Torres, P., and Morgado-Bernal, I. (1989). Correlations between paradoxical sleep and shuttle-box conditioning in rats. Behav. Neurosci. 103, 984-990. doi: 10.1037//0735-7044.103. 5.984

Prince, T. M., Wimmer, M., Choi, J., Havekes, R., Aton, S., and Abel, T. (2014). Sleep deprivation during a specific 3-hour time window post-training impairs hippocampal synaptic plasticity and memory. Neurobiol. Learn. Mem. 109, 122 130. doi: 10.1016/j.nlm.2013.11.021

Ruskin, D. N., and Lahoste, G. J. (2009). Reduced-volume cues effectively support fear conditioning despite sleep deprivation. Physiol. Behav. 96, 64-66. doi: 10. 1016/j.physbeh.2008.08.014

Ruskin, D. N., Liu, C., Dunn, K. E., Bazan, N. G., and Lahoste, G. J. (2004). Sleep deprivation impairs hippocampus-mediated contextual learning but not amygdala-mediated cued learning in rats. Eur. J. Neurosci. 19, 3121-3124. doi: 10.1111/j.0953-816x.2004.03426.x

Silva, R. H., Chehin, A. B., Kameda, S. R., Takatsu-Coleman, A. L., Abílio, V. C., Tufik, S., et al. (2004). Effects of pre- or post-training paradoxical sleep deprivation on two animal models of learning and memory in mice. Neurobiol. Learn. Mem. 82, 90-98. doi: 10.1016/j.nlm.2004.04.005

Silvestri, A. J. (2005). REM sleep deprivation affects extinction of cued but not contextual fear conditioning. Physiol. Behav. 84, 343-349. doi: 10.1016/j.physbeh. 2004.11.011

Silvestri, A. J., and Root, D. H. (2008). Effects of REM deprivation and an NMDA agonist on the extinction of conditioned fear. Physiol. Behav. 93, 274-281. doi: 10.1016/j.physbeh.2007.08.020

Smith, C. (1996). Sleep states, memory processes and synaptic plasticity. Behav. Brain Res. 78, 49-56. doi: 10.1016/0166-4328(95)00218-9

Smith, C. T., Conway, J. M., and Rose, G. M. (1998). Brief paradoxical sleep deprivation impairs reference, but not working, memory in the radial arm maze task. Neurobiol. Learn. Mem. 69, 211-217. doi: 10.1006/nlme.1997. 3809

Smith, C., and Lapp, L. (1986). Prolonged increases in both PS and number of REMS following a shuttle avoidance task. Physiol. Behav. 36, 1053-1057. doi: 10. 1016/0031-9384(86)90479-8

Smith, C. T., Nixon, M. R., and Nader, R. S. (2004). Posttraining increases in REM sleep intensity implicate REM sleep in memory processing and provide a biological marker of learning potential. Learn. Mem. 11, 714-719. doi: 10. 1101/lm.74904

Smith, C., and Rose, G. M. (1996). Evidence for a paradoxical sleep window for place learning in the Morris water maze. Physiol. Behav. 59, 93-97. doi: 10. 1016/0031-9384(95)02054-3

Smith, C., and Rose, G. M. (1997). Posttraining paradoxical sleep in rats is increased after spatial learning in the Morris water maze. Behav. Neurosci. 111, 1197-1204. doi: 10.1037//0735-7044.111.6.1197

Smith, C., Young, J., and Young, W. (1980). Prolonged increases in paradoxical sleep during and after avoidance-task acquisition. Sleep 3, 67-81.

Stern, W. C. (1971). Acquisition impairments following rapid eye movement sleep deprivation in rats. Physiol. Behav. 7, 345-352. doi: 10.1016/00319384(71)90312-x

Stickgold, R., Hobson, J. A., Fosse, R., and Fosse, M. (2001). Sleep, learning and dreams: off-line memory reprocessing. Science 294, 1052-1057. doi: 10. 1126/science. 1063530

Suchecki, D., Tiba, P., and Tufik, S. (2002). Hormonal and behavioural responses of paradoxical sleep-deprived rats to the elevated plus maze. J. Neuroendocrinol. 14, 549-554. doi: 10.1046/j.1365-2826.2002.00812.x

Suchecki, D., and Tufik, S. (2000). Social stability attenuates the stress in the modified multiple platform method for paradoxical sleep deprivation in the rat. Physiol. Behav. 68, 309-316. doi: 10.1016/s0031-9384(99)00181-x

Tartar, J. L., Ward, C. P., Cordeira, J. W., Legare, S. L., Blanchette, A. J., Mccarley, R. W., et al. (2009). Experimental sleep fragmentation and sleep deprivation in rats increases exploration in an open field test of anxiety while increasing plasma corticosterone levels. Behav. Brain Res. 197, 450-453. doi: 10.1016/j.bbr.2008. 08.035

Tiba, P. A., Oliveira, M. G., Rossi, V. C., Tufik, S., and Suchecki, D. (2008). Glucocorticoids are not responsible for paradoxical sleep deprivation-induced memory impairments. Sleep 31, 505-515. 
Walker, M. P., and Stickgold, R. (2004). Sleep-dependent learning and memory consolidation. Neuron 44, 121-133. doi: 10.1016/j.neuron.2004. 08.031

Walker, M. P., and Stickgold, R. (2005). It's practice, with sleep, that makes perfect: implications of sleep-dependent learning and plasticity for skill performance. Clin. Sports Med. 24, 301-317, ix. doi: 10.1016/j.csm.2004.11.002

Walker, M. P., and Van Der Helm, E. (2009). Overnight therapy? The role of sleep in emotional brain processing. Psychol. Bull. 135, 731-748. doi: 10.1037/a00 16570

Conflict of Interest Statement: The authors declare that the research was conducted in the absence of any commercial or financial relationships that could be construed as a potential conflict of interest.
Received: 11 August 2014; accepted: 20 October 2014; published online: 07 November 2014.

Citation: Rossi VC, Tiba PA, Moreira KDM, Ferreira TL, Oliveira MGM and Suchecki $D$ (2014) Effects of sleep deprivation on different phases of memory in the rat: dissociation between contextual and tone fear conditioning tasks. Front. Behav. Neurosci. 8:389. doi: 10.3389/fnbeh.2014.00389

This article was submitted to the journal Frontiers in Behavioral Neuroscience. Copyright (c) 2014 Rossi, Tiba, Moreira, Ferreira, Oliveira and Suchecki. This is an open-access article distributed under the terms of the Creative Commons Attribution License (CC BY). The use, distribution and reproduction in other forums is permitted, provided the original author(s) or licensor are credited and that the original publication in this journal is cited, in accordance with accepted academic practice. No use, distribution or reproduction is permitted which does not comply with these terms. 